\title{
The Role of Patient Education in Total Knee Arthroplasty: a new methodology for developing countries: a randomized controlled trial
}

Marco Antonio Percope de Andrade ( $\triangle$ mapa.bhz@terra.com.br)

Universidade Federal de Minas Gerais Faculdade de Ciencias Economicas https://orcid.org/00000001-6284-213X

Guilherme Moreira Abreu e Silva

Universidade Federal de Minas Gerais

Tulio Vinicius Oliveira Campos

Universidade Federal de Minas Gerais

David Guen Kasuya Barbosa MD

Universidade Federal de Minas Gerais

Danilo Silva Leite

Universidade Federal de Minas Gerais Faculdade de Ciencias Economicas

Marcus Vinicius Teodoro Rezende

Universidade Federal de Minas Gerais

Tauam Filipe Galo Magalhães

Universidade Federal de Minas Gerais

Felipe Maciel Santos

Universidade Federal de Minas Gerais Faculdade de Ciencias Economicas

\section{Research article}

Keywords: Arthroplasty, Knee Replacement, Osteoarthritis, Patient Education

Posted Date: January 20th, 2021

DOI: https://doi.org/10.21203/rs.3.rs-29921/v2

License: (c) (1) This work is licensed under a Creative Commons Attribution 4.0 International License.

Read Full License 


\section{Abstract}

Background: The illiteracy index is high in public hospitals of developing countries,. We established a method in which patients are instructed before total knee arthroplasty (TKA) in a differentiated way without the necessity of reading any self-orientation.

Methods: We developed a multidisciplinary approach to improve patient education in TKA comprising of a differentiated orientation conducted by an orthopedic surgeon, a nurse and a physiotherapist. It consists of standardized lectures regarding on pre, intra and post-operative issues in a randomized controlled trial of 79 consecutive patients undergoing primary TKA. Thirty-four patients received the standard education (control group) and 45 patients received the differentiated education (intervention group). The patients were evaluated during at least six months.

Results: After a 6-month follow-up period, the Short Form Health Survey (SF-36), the Western Ontario and McMaster Universities Osteoarthritis Index (WOMAC), the visual analogue pain scale (VAS) and knee range-of-motion (ROM) improved significantly in both groups. Range-of-motion was better in the intervention group (mean and SD - $106.9 \pm 5.7$ versus $92.5 \pm 12.1$ degrees, $p=0.02$ ). Moreover, walk ability (more than 400 meters) was better in the intervention group compared with the control group ( $97.4 \%$ versus $72.4 \%, p=0.003$ ). In the intervention and control groups, respectively, $10.5 \%$ and $31 \%$ of patients reported the need for some walking devices $(p=0.03)$.

Conclusions: A differentiated educational program with a multidisciplinary team had a positive impact on functional outcomes, improving ROM and walk ability of patients undergoing TKA in a short-term evaluation.

\section{Background}

Osteoarthritis $(\mathrm{OA})$ is a chronic and potentially disabling condition related to complex etiology. Its incidence has increased in the last few years, mainly due to population aging and an increased rate of obesity and sports injuries (1-3). Imbalance between anabolic and catabolic pathways usually leads to gradual cartilage damage (3). During this process, patients might experience functional impairment, pain and mechanical symptoms, which can impact their quality of life (4).

The knee is the second joint most affected by OA, i.e. approximately $37 \%$ of all patients seek medical assistance (5). Moreover, social and mental distress can be related to knee OA, impacting outcome negatively (6). Usually surgical treatment is recommended when conservative measures have failed (5). Total knee arthroplasty (TKA) has proved to be a successful treatment with predictable results (7-9). However, up to $20 \%$ of patients after their TKA complain of residual pain, functional impairment or subjective dissatisfaction (9). Mismatch between medical and patient expectations is one of the possible causes leading to dissatisfaction after TKA. Therefore, educating patients about the disease and the treatment process is an important measure in health assistance. 
Patient education refers to any intervention delivered during health assistance which aims to improve knowledge, health behaviors and health outcomes. As patients often forget verbal orientation, written and illustrated materials are effective in maximising knowledge and adhesion to treatment. However the majority of patients in public hospitals of developing countries are illiterate, which hinders this process. The recommended content of education varies across settings, but should comprise of discussion about pre-operative procedures, important surgical points, post-operative care, potential stressful scenarios, potential complications, pain management, discharge criteria and also postoperative rehabilitation (10). Although this pre-operative education seems obvious and embedded in the consent process, recent data questioned its efficacy to improve postoperative functional scores (11) Moreover, when treating illiterate patients, a reinforced educational approach should be applied. Our hypothesis is that a new educational pre-operative approach would improve the TKA results. The purpose of this study is to compare the clinical outcomes for two groups of patients who received different educational approaches before TKA. The groups were compared for function and clinical data.

\section{Methods}

A prospective randomized clinical trial was conducted from November 2017 to July 2018. An institutional review board approval from the Ethics Committee of the Universidade Federal de Minas Gerais was obtained for our research protocol to prospective data acquisition (CAAE:11677714.4.0000.5149), and an informed consent was signed by all the participants or one person responsible for them.

The sample size was calculated to test the hypothesis that a differentiated education would increase post-operative range-of-motion (ROM) of the knee joint (primary outcome). Trying to detect a difference of 10 degrees in ROM of the knee joint between groups and based on a power test of $80 \%$ and a confidence interval of $5 \%$ after assuming a possible $10 \%$ lost in follow-up, 15 patients in each group were considered the minimum number of participants.

Secondary outcomes were:

- the Short Form Health Survey (SF-36), which includes questions about patients general health

- the Western Ontario and McMaster Universities Osteoarthritis Index (WOMAC) - results range from 0 to 96 . It evaluates pain intensity (0-20), stiffness (0-8) and function (0-68)

- the visual analogue pain scale (VAS) - ranging from 0 to 10

- the distance the patients were able to walk and in the last follow-up if they were able to walk 400 meters or more

- the capacity to climb and descend stairs.

All variables were collected in the preoperative appointment which occurred at least 2 weeks before surgery and in the post-operative evaluations. 
Inclusion criteria: patients with unilateral symptomatic primary or secondary OA of the knee, older than 45 years, with indication of a primary TKA, who signed the informed consent form. Clinical examination and functional tests were applied to all participants by two investigators (DGKB, DSL).

Patients were then randomized into two groups: differentiated orientation or usual orientation. Randomization comprised choosing one of two closed envelopes with the words "usual" or "differentiated", referring to the approach it would be done.

Intervention group (45 patients): after medical appointment, patients received a new multidisciplinary reinforced orientation that included scheduled sessions of lectures done by an orthopedic surgeon, a nurse and a physiotherapist concerning the pre and post-operative care, pain management, rehabilitation exercises to gain ROM, the basic steps of the surgical procedure and the importance of walking with a walker. This intervention was done 2 weeks before surgery during office consultation. Patients were invited to attend a collective oral session, which included a standardized sequence of slides and images regarding the pre-operative preparation, the procedure itself and the rehabilitation with 1) a 15-minute lecture done by a nurse, 2) a 15-minute lecture done by a physiotherapist and 3) a 15-minute lecture done by an orthopedic surgeon, followed by additional time for patients to ask questions. Researchers also provided a phone contact number in case patients had further questions. The cost of this intervention method is low, which turns it feasible to be applied more widely (FIGURE 1).

Control group (34 patients): patients were oriented during conventional medical appointments, in which any doubts were clarified. A phone contact of researchers was provided for the patients.

The difference in the number of participants between groups was due to the study closure before 50 patients in each group were reached, considering that the minimal number to confirm our hypothesis was 15 patients.

The post-operative clinical and functional evaluation was performed by three blinded investigators (MVTR, TFGM, FSM). The study was conducted at the Orthopaedic Department of the Federal University of Minas Gerais - Brazil.

Seventy-nine patients were eligible for the study. Twelve were excluded during follow-up - 5 in the control group and 7 in the intervention group: one patient died (myocardial infarct), three patients had periprosthetic joint infection and eight did not return for control and were excluded. After exclusions, the control group and the intervention group remained with 29 and 38 patients, respectively (FIGURE 2).

All patients were operated on in the same Institution by three different surgeons (MAPA, GMAS, TVOC) who followed the same protocol. Patients received a peridural anesthesia with bupivacaine $(0,5 \%)$ and intravenous sedation (Diprovan ${ }^{\circledR}$ AstraZeneca). Tourniquet was applied in all cases set to 300 $\mathrm{mmHg}$. Primary TKA was performed through a classical medial arthrotomy with patellar eversion. A cruciate retained implant (Nexgen ${ }^{\circledR}$ Zimmer - Warsaw, IN), with patellar substitution, fixed with a non- 
impregnated antibiotic cement was used in all cases. Postoperative multimodal pain control protocol was made in both groups and started on the same day of the procedure. It consisted of scheduled 1) acetaminophen $133 \mathrm{mg}$ every 6 hours, 2) metamizole $500 \mathrm{mg}$ every 6 hours, 3) tramadol $50 \mathrm{mg}$ every 8 hours for 24 hours and 4) morphine 2 to $6 \mathrm{mg}$ every 4 hours, as needed. Patients were encouraged to start early ROM and weight bearing with a walker on the first day. Patients were discharged from hospital on day 2 or 3 and were evaluated in 15 days, 1 month, 3 months and 6 months after surgery and in each session, the scores and the VAS were applied, ROM was evaluated with a calibrated goniometer, and specific questions such as the use of walker or cane, their ability in climbing or descending stairs and the distance they were able to walk were asked.

Statistical analysis was performed to determine statistically significant differences between the 2 groups $(p<0.05)$, using appropriate software ( $G^{*}$ Power Version 3.1.9.2) and included analysis of distribution by Zolmogorov-Smirnov test. Independent T-test was used to analyze numerical, continuous and normally distributed variables. Qui-square test was used for categorical data. No crossover was observed, so intention-to-treat analysis was not performed.

\section{Results}

The two groups were comparable on demographics, clinical and functional tests (TABLE 1). After a 6month follow-up period, VAS, WOMAC and SF-36 scores significantly improved in both groups, and no difference was observed between groups. Knee range-of-motion was higher in the intervention group (mean flexion $106.9 \pm 5.7$ versus $92.5 \pm 12.1^{\circ}, \mathrm{p}=0.02$ ). Moreover, at 6 months, $97.4 \%$ of patients in the intervention group and $72.4 \%$ of patients in the control groups reported an improvement in their ability to walk more than 400 meters after surgery $(p=0.003)$. At six month follow-up-period, walk assistance was needed in $10.5 \%$ of the patients in the intervention group and in $31 \%$ in the control group $(p=0.03)$ (TABLE 2). No significant missing data was observed during this short-term study.

\section{Discussion}

This study showed that additional education improves functional results after TKA. Studied parameters were applied differently to both groups with no additional costs. The same infrastructure and health team was utilized in the intervention method. As a result of this intervention, patients who received a reinforced orientation protocol achieved better ROM and could walk more frequently without assistance. Regarding postoperative functional scores and pain, no difference was observed between groups.

Achieving good results after total knee replacement depends on many factors, such as patient selection, implant design, surgical technique and postoperative care (12). However, more than $20 \%$ of patients can still have some dissatisfaction after the procedure $(13,14)$. It is well known that ROM has correlation with better functional scores after TKA (15). 
Biomechanical studies showed that patients require at least $83^{\circ}$ of flexion to climb stairs, $100^{\circ}$ to descend stairs and $67^{\circ}$ to walk normally (16). Ritter et al. (15) observed worse functional outcomes in patients with less than 118 degrees of flexion. Kotani et al. (17) noted that more than $110^{\circ}$ of knee flexion would improve daily living activities. Thereby, a method that could help improving range of motion might favorably impact TKA outcomes.

On the other hand, the role of patient education was questioned due to low-quality evidence of available data (11), but these findings do not invalidate its use and the present study confirms its importance.

ROM evaluation as an independent predictor in TKA had not been clearly studied. Two studies depicted no difference in ROM between oriented and non-oriented patients after hip replacement $(18,19)$. Our study shows that a new reinforced education, with a team approach improved ROM when compared to the traditional orientation method. This reinforced education would avoid oversights during orientation by improving the understanding of the patient about the procedure. Al-Rub et al. (20) in a cohort study showed that $85 \%$ of patients did not know the composition of the implant and only $39 \%$ of patients had received advice about dental work after arthroplasty. Educating patients before surgery leads to decreased anxiety, which can be expressed by better ROM and walk ability after surgery (21). Our method may be applied on illiterate patients with a positive effect. Ayers et al. (22) showed that more than $30 \%$ of patients going through TKA have depression or anxiety symptoms, which can be minimized by better orientation. Illiteracy is still highly predominant in developing countries and therefore booklets do not prove very effective. The proposed new approach for illiterate patients is easy to apply, has low cost and leads to positive outcomes.

There are limitations in this study and a follow-up evaluation for a short period of time ( 6 months) can be considered a point of weakness. However, ROM and walk capacity after 6 months can clearly predict long-term results $(23,24)$. Subsequently, function improvement is less likely without some medical intervention (manipulation under anesthesia, arthrolysis or revision) (25).

\section{Conclusion}

After a mean 6-month period, a new reinforced orientation method comprising multidisciplinary oral presentations with instructional lectures improved the final ROM and also the walking capacity after TKA. This method can be applied to illiterate patients, commonplace in public hospitals of developing countries.

\section{Abbreviations}

TKA: total knee arthroplasty; SF-36: Medical Study 36-item Short-Form Health Survey; WOMAC: Western Ontario and McMaster Universities Osteoarthritis Index; ROM: range of motion; OA: osteoarthritis; VAS: visual analog scale 


\section{Declarations}

The authors declare no conflict of interest regarding this research

\section{Acknowledgements}

No further acknowledgements

\section{Authors' contributions}

MAPA conceptualized the study and design. DGKB, DSL, MVTR, TFGM and FSM recruited the participants and collected the data. MAPA, GMAS and TVOC prepared the first draft of the manuscript and all authors contributed to writing, as well as review and approval of the final version of the manuscript.

\section{Availability of data and materials}

The authors have full access to all dataset in the study and assume final responsibility for the publication. The data used and analyzed during the current study are available through the corresponding author upon reasonable request.

\section{Ethics approval and consent to participate.}

All procedures performed in studies involving human participants were in accordance with the ethical standards of the institutional and/or national research committee and with the 1964 Helsinki declaration and its later amendments. The consent obtained from study participants was written and approved by the Ethics Committee. An institutional review board approval from the Ethics Committee of the Universidade Federal de Minas Gerais was obtained for our research protocol to prospective data acquisition of 39 patients undergoing TKA (CAAE:11677714.4.0000.5149), and an informed consent was signed by all the participants or one person responsible for them.

\section{Consent for publication}

Not Applicable

\section{Competing interests}

Authors declare that they do not have any financial and non-financial competing interests.

\section{Funding}

No funding was obtained for this study

\section{Authors details}

All authors are from the Departamento do Aparelho Locomotor - Faculdade de Medicina - Universidade Federal de Minas Gerais 


\section{References}

1. Buckwalter JA. Osteoarthritis and articular cartilage use, disuse, and abuse: experimental studies. J Rheumatol Suppl. 1995;43:13-5.

2. Neogi T, Zhang Y. Epidemiology of osteoarthritis. Rheum Dis Clin North Am. 2013;39(1):1-19.

3. Murphy L, Schwartz TA, Helmick CG, Renner JB, Tudor G, Koch G, et al. Lifetime risk of symptomatic knee osteoarthritis. Arthritis Rheum. 2008;59(9):1207-13.

4. Arden N, Nevitt MC. Osteoarthritis: epidemiology. Best Pract Res Clin Rheumatol. 2006;20(1):3-25.

5. Senna ER, De Barros AL, Silva EO, Costa IF, Pereira LV, Ciconelli RM, et al. Prevalence of rheumatic diseases in Brazil: a study using the COPCORD approach. J Rheumatol. 2004;31(3):594-7.

6. Utrillas-Compaired A, De la Torre-Escuredo BJ, Tebar-Martínez AJ, Asúnsolo-Del Barco Á. Does preoperative psychologic distress influence pain, function, and quality of life after TKA? Clin Orthop Relat Res. 2014;472(8):2457-65.

7. Pagnano MW, McLamb LA, Trousdale RT. Total knee arthroplasty for patients 90 years of age and older. Clin Orthop Relat Res. 2004(418):179-83.

8. Ethgen $O$, Bruyère $O$, Richy F, Dardennes $C$, Reginster JY. Health-related quality of life in total hip and total knee arthroplasty. A qualitative and systematic review of the literature. J Bone Joint Surg Am. 2004;86-A(5):963-74.

9. Shan L, Shan B, Suzuki A, Nouh F, Saxena A. Intermediate and long-term quality of life after total knee replacement: a systematic review and meta-analysis. J Bone Joint Surg Am. 2015;97(2):156-68.

10. Louw A, Diener I, Butler DS, Puentedura EJ. Preoperative education addressing postoperative pain in total joint arthroplasty: review of content and educational delivery methods. Physiother Theory Pract. 2013;29(3):175-94.

11. McDonald S, Page MJ, Beringer K, Wasiak J, Sprowson A. Preoperative education for hip or knee replacement. Cochrane Database Syst Rev. 2014;5:CD003526.

12. Sanz-Ruiz P, Carbo-Laso E, Alonso-Polo B, Matas-Diez JA, Vaquero-Martín J. Does a new implant design with more physiological kinematics provide better results after knee arthroplasty? Knee. 2016.

13. Noble PC, Conditt MA, Cook KF, Mathis KB. The John Insall Award: Patient expectations affect satisfaction with total knee arthroplasty. Clin Orthop Relat Res. 2006;452:35-43.

14. Bourne RB, Chesworth BM, Davis AM, Mahomed NN, Charron KD. Patient satisfaction after total knee arthroplasty: who is satisfied and who is not? Clin Orthop Relat Res. 2010;468(1):57-63.

15. Ritter MA, Lutgring JD, Davis KE, Berend ME. The effect of postoperative range of motion on functional activities after posterior cruciate-retaining total knee arthroplasty. J Bone Joint Surg Am. 2008;90(4):777-84.

16. Laubenthal KN, Smidt GL, Kettelkamp DB. A quantitative analysis of knee motion during activities of daily living. Phys Ther. 1972;52(1):34-43. 
17. Kotani A, Yonekura A, Bourne RB. Factors influencing range of motion after contemporary total knee arthroplasty. J Arthroplasty. 2005;20(7):850-6.

18. Gocen Z, Sen A, Unver B, Karatosun V, Gunal I. The effect of preoperative physiotherapy and education on the outcome of total hip replacement: a prospective randomized controlled trial. Clin Rehabil. 2004;18(4):353-8.

19. Vukomanović A, Popović Z, Durović A, Krstić L. The effects of short-term preoperative physical therapy and education on early functional recovery of patients younger than 70 undergoing total hip arthroplasty. Vojnosanit Pregl. 2008;65(4):291-7.

20. Abu Al-Rub Z, Hussaini M, Gerrand $\mathrm{CH}$. What do patients know about their joint replacement implants? Scott Med J. 2014;59(3):158-61.

21. Demir F, Ozsaker E, Ilce AO. The quality and suitability of written educational materials for patients*. J Clin Nurs. 2008;17(2):259-65.

22. Ayers DC, Franklin PD, Trief PM, Ploutz-Snyder R, Freund D. Psychological attributes of preoperative total joint replacement patients: implications for optimal physical outcome. J Arthroplasty. 2004;19(7 Suppl 2):125-30.

23. Nilsdotter AK, Toksvig-Larsen S, Roos EM. A 5 year prospective study of patient-relevant outcomes after total knee replacement. Osteoarthritis Cartilage. 2009;17(5):601-6.

24. Papakostidou I, Dailiana ZH, Papapolychroniou T, Liaropoulos L, Zintzaras E, Karachalios TS, et al. Factors affecting the quality of life after total knee arthroplasties: a prospective study. BMC Musculoskelet Disord. 2012;13:116.

25. Fitzgerald JD, Orav EJ, Lee TH, Marcantonio ER, Poss R, Goldman L, et al. Patient quality of life during the 12 months following joint replacement surgery. Arthritis Rheum. 2004;51(1):100-9.

\section{Tables}

Due to technical limitations, Table 1 and Table 2 are only available as a download in the supplementary files section.

\section{Figures}




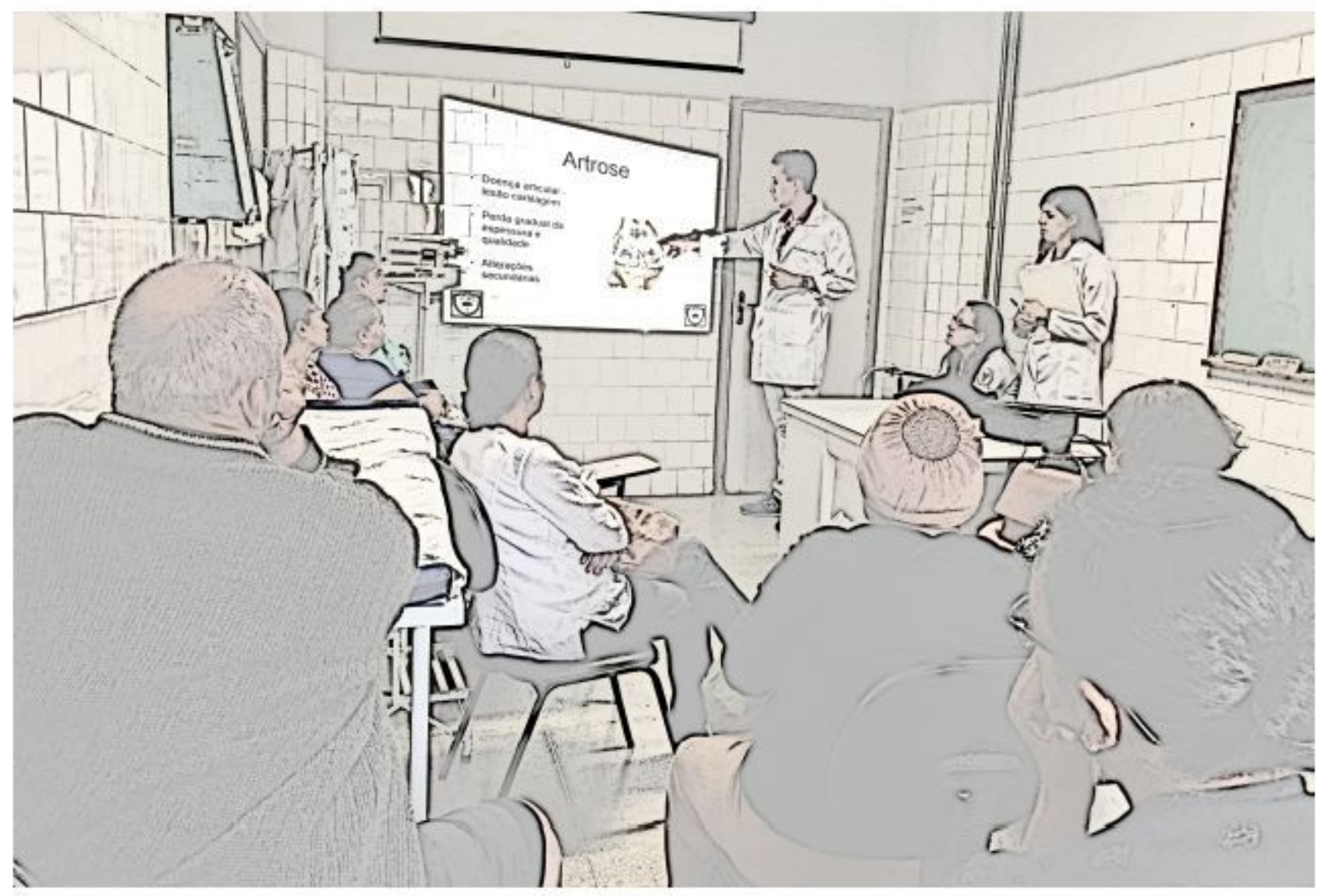

Figure 1

Eligible patients groups attending a new reinforced educational method session that consisted on illustration based and interactive discussion with TKA candidates 


\section{CONSORT \\ TRANSPARENT REPORTING of TRIALS}

A new educational method before total knee arthroplasty to be applied in developing countries: a randomized controlled trial

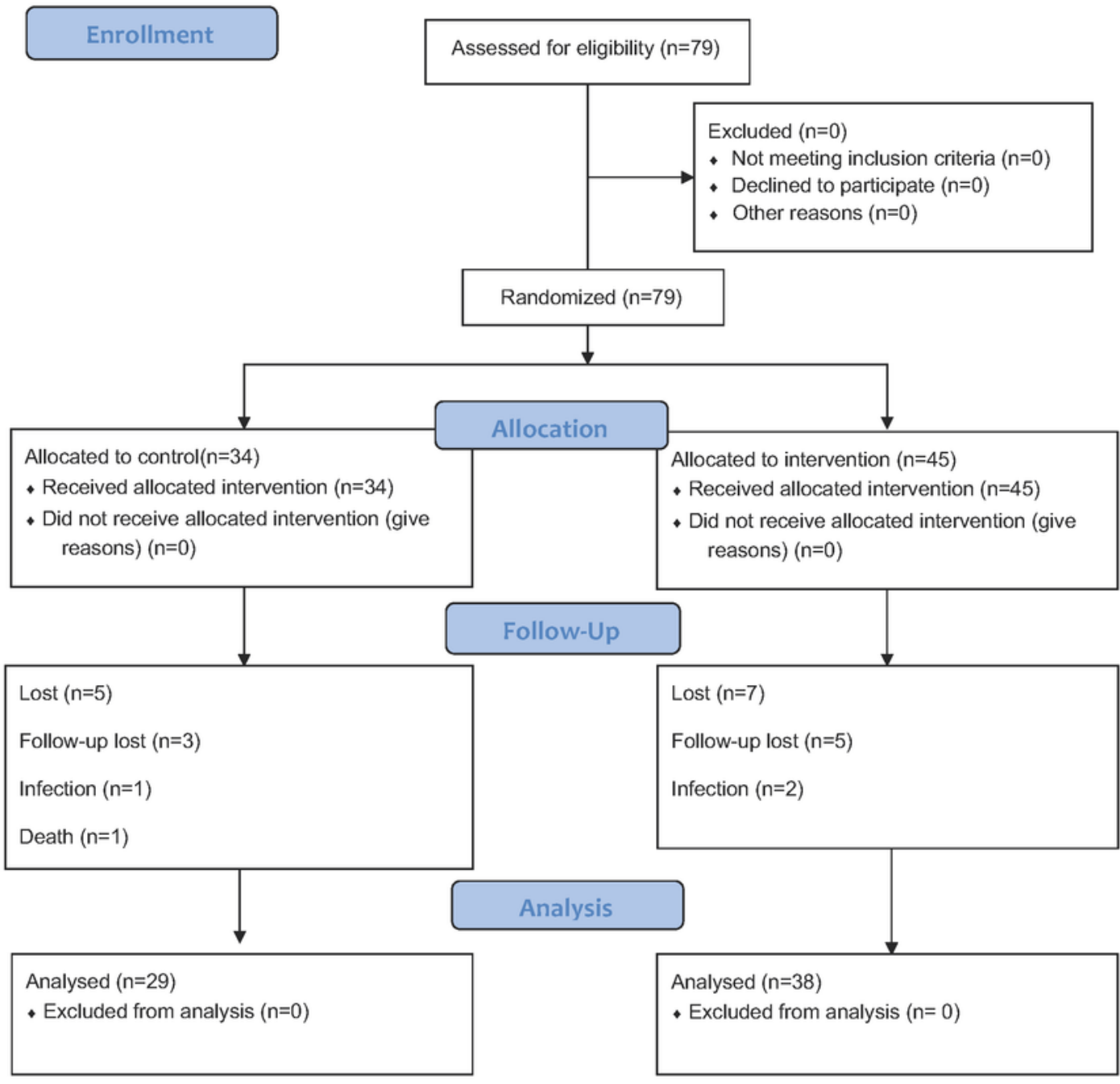

Figure 2

Patient allocation fluxogram

\section{Supplementary Files}

This is a list of supplementary files associated with this preprint. Click to download. 
- Table1.pdf

- Table2.pdf

- Rawdata1.xlsx

Page 12/12 